\title{
Primer registro de leucismo en el género Coendou Lacépède, 1799 (Rodentia: Erethizontidae)
}

\author{
Julio C. Romero-Briceño*iD, José A. González-Carcacíaº(iD) \\ 1 Laboratorio de Producción Farmacéutica Veterinaria VIRBAC-CENTROVET, Avenida Salomón Sack 255, Santiago de Chile, \\ Chile. \\ 2 Instituto Venezolano de Investigaciones Científicas IVIC. Centro de Ecología. Laboratorio de Biología de Organismos. \\ Carretera Panamericana, Km. 11, Caracas 1020A, Estado Miranda, Venezuela. \\ *Correspondencia: julio.cactus@gmail.com
}

\section{Resumen}

Se reporta por primera vez un caso de leucismo en la especie Coendou prehensilis. El individuo fue hallado de forma fortuita en la localidad Monumento Virgen de la Paz (Trujillo), una zona de bosque perturbado en la región de Los Andes en Venezuela.

Palabras clave: Melanina, Mamífero Neotropical, Puercoespín, Estado Trujillo, Venezuela.

\section{Abstract}

A case of leucism in the Coendou prehensilis species is reported for the first time. The individual was found by chance in the Virgen de la Paz Monument (Trujillo), a disturbed forest area in the Andes region of Venezuela.

Key words: Melanin, Neotropical Mammal, Porcupine.

El leucismo es un trastorno cromático no letal de origen genético (Beerman et al. 2004; Velandia-Perilla et al. 2013), que no es frecuente en mamíferos (Caro 2005; Abreu et al. 2013; Romero \& Tirira 2017) y que resulta en la ausencia de melanina en animales que normalmente son pigmentados (Lawrence 1999; Álvarez de Villar et al. 2007), pero sin extenderse hasta el iris ocular (Acevedo \& Aguayo 2008; Abreu et al. 2013; Lucati \& LópezBaucells 2016). Los individuos que presentan esta característica fenotípica probablemente sean seleccionados negativamente en sus poblaciones, debido a la dificultad de interaccionar con sus congéneres y a la alta detectabilidad ante sus depredadores, lo que trae como consecuencia una baja supervivencia y éxito reproductivo (Miller 2005; MartínezCoronel et al. 2013; Romero-Briceño 2018). Sin embargo, el hallazgo de individuos adultos con esta condición, hace parecer que las consecuencias negativas mencionadas anteriormente no impiden su desarrollo (Camargo et al. 2014; Arriaga-Flores et al. 2016; Puig et al. 2017). 
Los roedores (Rodentia) son el orden más diverso de mamíferos en el mundo con 2.482 especies (Wilson et al. 2017). En Sudamérica existen alrededor de 642 especies pertenecientes a este grupo (Patton et al. 2015). De estas, la coloración anómala del tipo leucismo ha sido reportado en solamente 13 especies que corresponden a ejemplares recolectados en Ecuador, Brasil y Chile (Brito \& Valdivieso-Bermeo 2016; Rubio \& Simonetti 2019; Ramírez-Jaramillo 2019). Sin embargo, hasta el momento en Venezuela han sido poco o nada documentados. En esta nota se documenta el primer registro de leucismo para el género Coendou y en general para especies de roedores en Venezuela.

Coendou prehensilis es una especie de roedor de mediano tamaño, ampliamente distribuido en Sudamérica, abarcando desde Colombia y Venezuela hasta Argentina entre 50 y 2.600 msnm (Woods \& Kilpatrick 2005; Voss 2011, 2015; Barthelmess 2016). Está presente en hábitats diversos, incluyendo bosques secos, húmedos, sabanas y zonas perturbadas (Voss et al. 2013; Voss 2015). Se encuentra en la categoría de Preocupación Menor de la Lista Roja de la IUCN (Marinho-Filho \& Emmons 2016).

El ejemplar reportado, es un adulto (HBL: $480 \mathrm{~mm}$ ) con el seno nasofrontal inflado característico de la especie. Se encontró el 10 de diciembre de 2014 alrededor de las 18 h00 en medio de un potrero ganadero del sector Monumento Virgen de la Paz (9,34708, -70,46258, WGS-84; 1.507 msnm), en la jurisdicción del municipio Trujillo del estado Trujillo. La región corresponde a la zona de vida de Bosque Seco Tropical (Ewel et al. 1976). Es el primer reporte de la especie para este estado, complementando los registros de localidades cercanas en los estados Lara, Barinas y Portuguesa. En esta zona C. prehensilis habitaría en simpatría con la especie C. pruinosus, sin embargo, en campo se diferencian por el tamaño, debido a que el primero es más grande (HBL 403-530 mm vs. 320-350 mm), además, el pelaje de C. pruinosus se compone de una mezcla de pelos y espinas que difiere con C. prehensilis en el grosor de las espinas y en el patrón de coloración (Linares 1998; Voss 2001; Ramírez-Chaves et al. 2016). El ejemplar con leucismo (Figura 1), presentó una coloración blanca en la mayor parte de las espinas dorsales y ventrales, así como en el resto del cuerpo; incluyendo pelos debajo de las espinas y alrededor del hocico. Los ojos presentaron iris y pupilas de color marrón oscuro. La cola y superficie dorsal de patas y garras tienen tonos que varían de marrón oscuro a casi negro. La coloración normal de C. prehensilis presenta espinas tricolores en la parte superior de la cabeza (blanco amarillento basalmente, negro en el medio y blanco o amarillo blanquecino distalmente). Pelos negros y dispersos ocultos debajo de espinas. Espinas alrededor de los ojos, hocico y mejillas blanquecino basal y distalmente, y negro en el medio. Pelos alrededor de la nariz amarronados. Vibrisas mystaciales, suboculares, supraorbitales, genales y submentales totalmente negras, espinas negras basalmente y blancas distalmente alrededor del trago (Figura 2) (Leite et al. 2011).

Los factores causales de los patrones de coloración atípicos en puercoespines neotropicales aún son inciertos (Romero et al. 2018). Existe poca información sobre la condición de individuos con leucismo bajo condiciones ambientales naturales. Por lo tanto, sería importante continuar con registros de este tipo, para comprender mejor sus implicaciones ecológicas. 


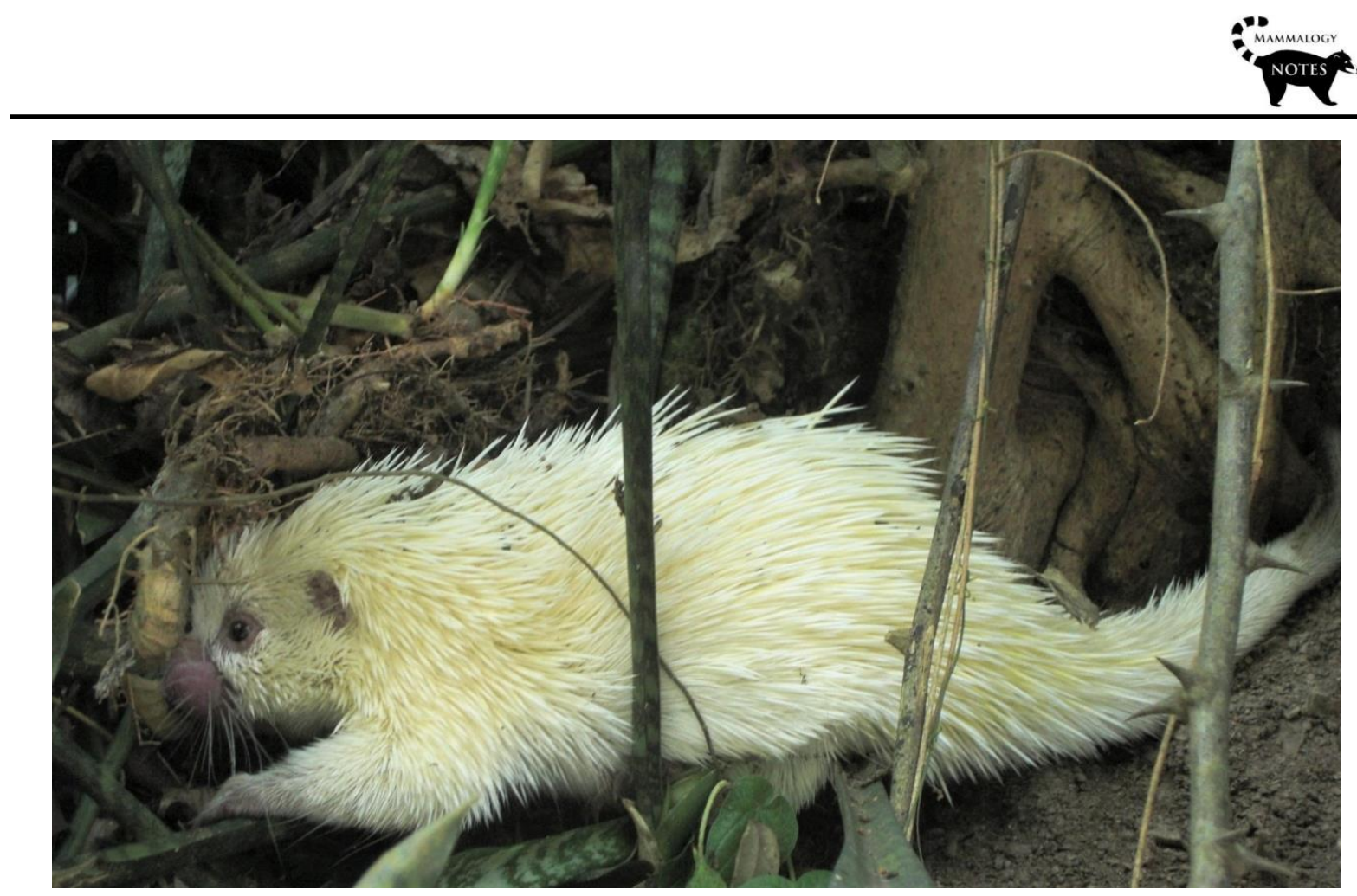

FIGURA 1. Ejemplar con leucismo, hallado en el sector Monumento Virgen de la Paz. Trujillo. El individuo fue liberado en el mismo punto de captura después de ser fotografiado y no mostró ninguna dificultad para moverse. Foto: Valter Goncalves.

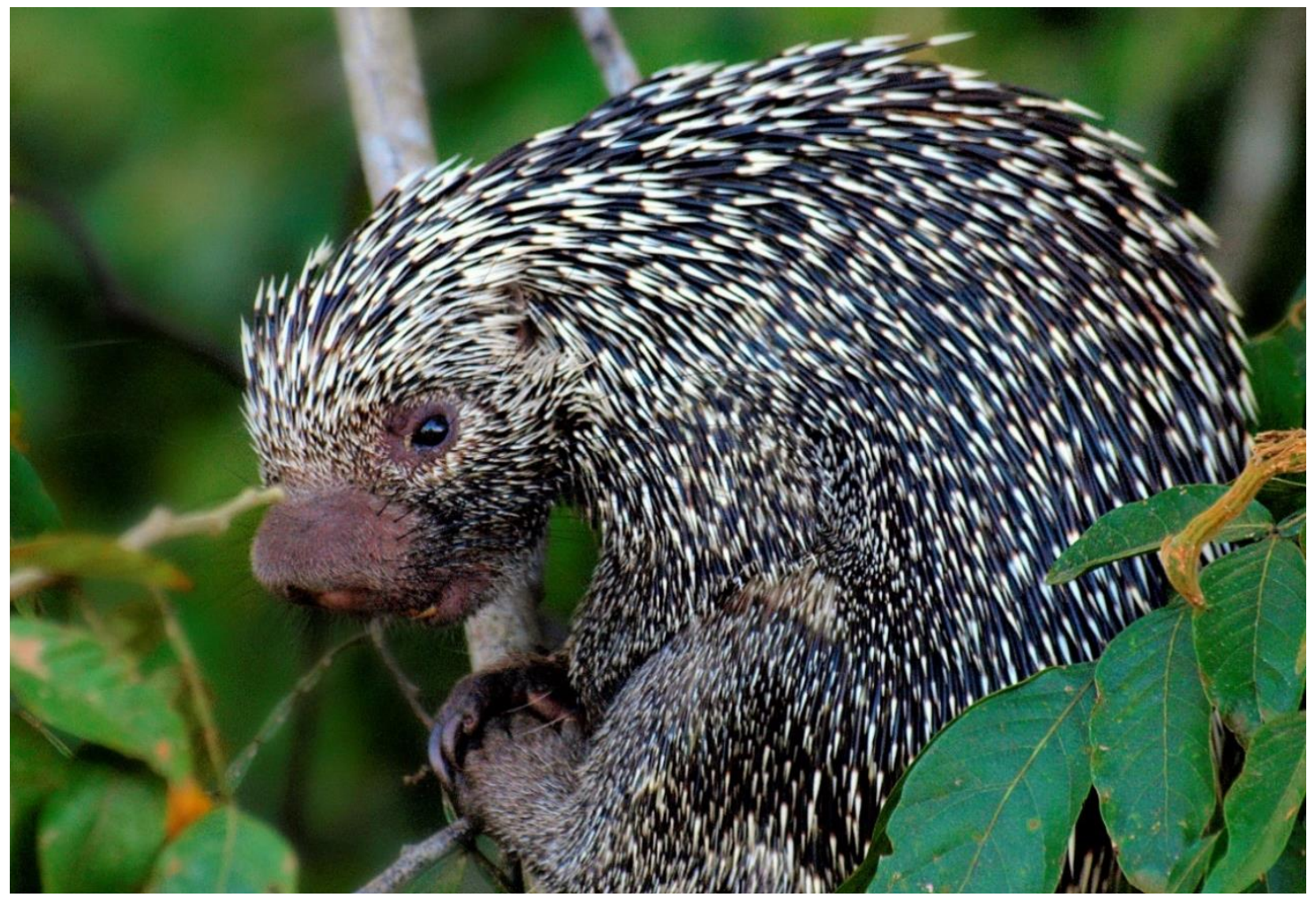

FIGURA 2. Coloración normal de C. prehensilis. (Reserva Forestal de Caparo, Estado Barinas). Foto: Diana Liz Duque Sandoval. 


\section{AGRADECIMIENTOS}

Estamos agradecidos con el amigo Valter Goncalves de la comunidad de Trujillo por su apoyo fotográfico durante el trabajo de campo, y con Carla Aranguren por ayudar al acceso a la bibliografía correspondiente. También a dos revisores anónimos que permitieron mejorar este manuscrito.

\section{REFERENCIAS}

Abreu MS, Machado R, Barbieri F, Freitas NS, Oliveira LR. 2013. Anomalous color in Neoptropical mammals: a review with new records for Didelphis sp. (Didelphidae, Didel-phimorphia) and Arctocephalus australis (Otariidae, Carnivora). Brazilian Journal of Biology 73:185-194. https:/ / doi.org/10.1590/S1519-69842013000100020

Acevedo J, Aguayo M. 2008. Leucistic South American sea Lion in Chile, with a review of anomalously color in otariids. Revista de Biología Marina y Oceanografia 23:413-417. http://dx.doi.org/10.4067/S0718-19572008000200017

Álvarez De Villar J, Álvarez T, Álvarez-Castañeda ST. 2007. Diccionario de Anatomía Comparada de Vertebrados. Instituto Politécnico Nacional. México, D.F.

Arriaga-Flores JC, Rodríguez-Ruíz ER, Gallo-Reynoso JP, Castro-Arellano I. 2016. Leucism in neotropical otters (Lontra longicaudis annectens) from Mexico. The Southwestern Naturalist 61: 63-68 https: / / doi.org/10.1894/0038-4909-61.1.63

Beerman F, Orlow SJ, Lamoreux ML, 2004. The Tyr (albino) locus of the laboratory mouse. Mammalian Genome 15:749-758. https:// doi.org/10.1007/s00335-004-4002-8

Barthelmess EL. 2016. Familia Erethizontidae. In: Wilson DE, Lacher TE, Mittermeier RA, editors. Handbook of Mammals of the World. Vol. 6. Lagomorfos y roedores: Lynx, Barcelona.

Brito J, Valdivieso-Bermeo K. 2016. First records of leucism in eight species of small mammals (Mammalia: Rodentia). Therya 7: 483-489. http:// dx.doi.org/10.12933/therya-16-408

Camargo I, Ríos E, Cornejo-Latorre E, Álvarez-Castañeda ST. 2014. First record of leucism in the genus Peromyscus (Mammalia: Rodentia). Western North American Naturalist. 74: 366-368. https:// doi.org/10.3398/064.074.0301

Caro T. 2005. The adaptive significance of coloration in mammals. Bioscience 55:125- 136. https:// doi.org/10.1641/0006-3568(2005)055[0125:TASOCI]2.0.CO;2

Ewel JJ, Madriz A, Tosi JA. 1976. Zonas de vida de Venezuela. Memoria explicativa sobre el mapa ecológico. Ministerio de Agricultura y Cría, FONAIAP, Caracas.

Lawrence E. 1999. Henderson's dictionary of biological terms. Pearson Prentice Hall. London.

Leite YLR, Caldera VJ, Loss AC, Costa LP, Melo ERA, Gadelha JR, Pontes ARM. 2011. Designation of a neotype for the Brazilian porcupine, Coendou prehensilis (Linnaeus, 1758). Zootaxa 2791: 30-40. https:/ / doi.org/10.11646/zootaxa.2791.1.2

Linares O. 1998. Mamíferos de Venezuela. Sociedad Conservacionista Audubon de Venezuela. Caracas, Venezuela.

Lucati F, López-Baucells A. 2016. Chromatic disorders in bats: a review of pigmentation anomalies and the misuse of terms to describe them. Mammal Review. 2:112-123. https:// doi.org/10.1111/mam.12083

Marinho-Filho J, Emmons L. 2016. Coendou prehensilis. The IUCN Red List of Threatened Species. 2016: e.T101228458A22214580.

2.RLTS.T101228458A22214580.en. Downloaded on 10 May 2020.

Martínez-Coronel M, Bautista R, Verona-Trejo MI. 2013. Albinismo platinado en Liomys pictus (Mammalia: Heteromyidae). Therya 4:641-645. http:// dx.doi.org/10.12933/therya-13-149

Miller JD. 2005. All about albinism. Missouri Conservationist 66: 5-7. 
Patton JL, Pardiñas UFJ, D'Elía G. 2015. Mammals of South America. Vol. 2. Rodents. The University of Chicago Press. Chicago.

Puig S, Videla F, Rosi MI, Seitz VP, Moreni J, Pérez M, Tobares R, Maldonado F, Martín S. 2017. First records of albine guanacos in the mountains of southern Andean Precordillera (Mendoza, Argentina). Multequina: Revista Latinoamericana de Recursos Naturales. 26:77-86.

Ramírez-Chaves HE, Suárez-Castro AF, Morales-Martínez DM, Vallejo-Pareja MC. 2016. Richness and distribution of porcupines (Erethizontidae: Coendou) from Colombia. Mammalia 80:181-191. https:// doi.org/10.1515/mammalia-2014-0158

Ramirez-Jaramillo SM. 2019. Un caso de leucismo parcial en la ardilla de cola roja Notosciurus granatensis (Rodentia: Sciuridae) en el Sur-oeste de Ecuador. Mammalogy Notes 5(2): 24-25. https://doi.org/10.47603/manovol5n2.24-25

Romero-Briceño JC. 2018. Un caso de xantismo en Chelonoidis carbonaria (Spix, 1984) (Testunides: Testudinidae) en los Llanos centrales de Venezuela. Memoria de la Fundación La Salle de Ciencias Naturales 184: 103-107.

Romero V, Recines-Márquez C, Brito J. 2018. A short review and worlwide list of wild albino rodents with the first report of albinism in Coendou rufescens (Rodentia: Erethizontidae). Mammalia 82: 509515. https:// doi.org/10.1515/mammalia-2017-0111

Romero V, Tirira D. 2017. Leucistic Antarctic fur seal (Arctocephalus gazella) at Robert Island, South Shetland Islands, Antarctica, with a note on colour morph nomenclature. Polar Biolology. 40: 1893-1897. https:// doi.org/10.1007/s00300-016-2069-9

Rubio AV, Simonetti JA. 2019. Partial and complete leucism in two Abroth-rix species (Rodentia: Cricetidae) from central Chile. Mammalia 83(1): 100-102. https:/ / doi.org/10.1515/mammalia-2017-0173

Velandia-Perilla J, Yusti-Muñoz AP, Sánchez-Martínez MA, Giraldo A. 2013. Leucismo en murciélagos de hoja nasal (Chiroptera: Phyllostomidae) de Colombia. Scientific Bulletin Museum Center Natural History Museum. 17(2):87-94.

Voss RS. 2011. Revisionary notes on Neotropical porcupines (Rodentia: Erethizontidae). 3. An annotated checklist of species of Coendou Lacépède, 1799. American Museum Novitates. 3720: 1-36.

Voss RS, Hubbard C, Jansa SA. 2013. Phylogenetic relationships of New World porcupines (Rodentia: Erethizontidae): implications for taxonomy, morphological evolution, and biogeography. American Museum Novitates 3769:1-36.

Voss RS 2015. Familia Erethizontidae Bonaparte, 1845. In: Patton JL, Pardiñas UFJ, D'Elía G, editors. Mammals of South America. The University of Chicago Press. p. 786-805.

Wilson DE, Lacher T, Mittermeier R. 2017. Handbook of the Mammals of the world. Vol. 7: Rodents II. Barcelona, Spain: Lynx Editions.

Woods CA, Kilpatrick CW. 2005. Suborden Hystricognathi. In: Wilson DE, Reeder DM, editors. Mammal species of the world: a taxonomic and geographic reference. 3a ed., John Hopkins University Press, Baltimore.p. 1538-1600

Editor: José F. González-Maya Recibido 2020-06-03 Revisado: 2020-07-01 Aceptado 2020-08-20 Publicado 2020-12-08 